\title{
Comparison of the effect of the intracameral lidocaine anesthesia and subconjunctival lidocaine anesthesia on the development of intraoperative floppy-iris syndrome in cataract surgery
}

\author{
(D)Tayfun Şahin ${ }^{1}$, ĐS Sücattin İlker Kocamış \\ ${ }^{1}$ Hitit University, Faculty of Medicine, Department of Ophthalmology, Çorum, Turkey \\ ${ }^{2}$ Sakarya Yenikent State Hospital, Department of Ophthalmology, Sakarya, Turkey
}

Cite this article as: Şahin T, Kocamış Sİ. Comparison of the effect of the intracameral lidocaine anesthesia and subconjunctival lidocaine anesthesia on the development of intraoperative floppy-iris syndrome in cataract surgery. J Health Sci Med 2021; 4(6): $761-765$.

\begin{abstract}
Aim: To evaluate the effect of intracameral lidocaine anesthesia (ILA) and subconjunctival lidocaine anesthesia (SLA) administered during cataract surgery on the development and prevalence of intraoperative floppy iris syndrome (IFIS).

Material and Method: The study involved the medical records of 86 cataract patients having no risk factors other than small pupil size for IFIS whom ILA and/or SLA were applied during phacoemulsification surgery. While 45 patients were administered intracameral lidocaine anesthesia ILA (1\%), 41 patients in the other group were administered subconjunctival lidocaine anesthesia (SLA) (2\%). Floppy iris syndrome findings such as iris billowing, iris incarceration in the wound site, or progressive myosis, if any, were recorded. The groups were compared in terms of the prevalence of IFIS development.

Results: While IFIS ratio was $33.7 \%$ in the ILA group, it was $17.1 \%$ in the SLA group ( $\mathrm{p}=0.084)$. Patients with smaller pupil diameter observed a higher IFIS rate ( when pupil diameter threshold value was $6.5 \mathrm{~mm} \mathrm{p}=0.011$ and $6 \mathrm{~mm} \mathrm{p}=0.009$ ).

Conclusion: During cataract surgery, surgeons should care for the development of IFIS in patients with small pupil diameters. However, the effect of intracameral lidocaine use on the development of IFIS has not been determined.
\end{abstract}

Keywords: Cataract, phacoemulsification, iris diseases, local anesthesia

\section{INTRODUCTION}

Cataract is the most common cause of blindness worldwide, and cataract surgery is one of the most frequently performed surgeries. Although many causes are counted in the etiology, the mechanisms of cataract formation are not fully elucidated. Today, the only treatment option is surgery. The preferred method in cataract surgery is phacoemulsification combined lens implantation due to reduced complication rates and high success rates (1). Patients could be administered general anesthesia or local anesthesia during cataract surgery. Local anesthesia could be in the form of topical, retrobulbar, peribulbar or sub-Tenon's blocks. Topical anesthesia could be combined with subconjunctival anesthesia or intracameral anesthesia to increase patient comfort. Unpreserved lidocaine (1\%) 0.3-0.5 mL could be used for this purpose in intracameral anesthesia $(2,3)$. Applicability of intracameral anesthesia is easy. This very commonly used anesthesia method yield good results with the right patient selection (4). Subconjunctival anesthesia could be applied in pterygium excision, conjunctiva surgeries, glaucoma and cataract surgeries (5).

Intraoperative floppy-iris syndrome (IFIS) is a condition associated with iris anomalies that might emerge during cataract surgery. Many factors are considered responsible for its etiology. IFIS could develop depending on the use of various systemic medications. The most important group among these is $\alpha$-receptor antagonists (6). IFIS could emerge in the forms of floppy iris stroma billowing with the liquid flow, the tendency of iris tissue to be prolapsed from the wound site, and progressive intraoperative pupillary constriction. IFIS was first defined in patients who used tamsulosin (7). Other studies conducted later confirmed that tamsulosin could cause IFIS $(8,9)$. It was also reported that apart from tamsulosin, IFIS 
could develop depending on $\alpha-1$ blockers, 5 - $\alpha$ reductase inhibitors, some herbal medicine (saw palmetto), and some antipsychotic medicine (10-14).

The complication ratio is reported to increase during surgery in the eyes with IFIS. Cases that developed IFIS could experience iris trauma due to unexpected iris prolapses and myosis, iris capture during phacoemulsification or irrigation, iridodialysis, hyphema, posterior capsule damage, and vitreous loss (15). Some studies report that complication ratios might not increase if the condition is predicted and patients receive operation from experienced surgeons (16). When IFIS develops during surgery, the use of intracameral epinephrine and iris hooks in tandem could enable to enlarge the pupil adequately and complete the surgery successfully (17). In addition, soft-shell viscoelastic technique, low liquid flow velocity, visco-adaptive viscoelastic use, pupil expansion rings, and staining the capsule with trypan blue are recommended when IFIS develops (18). This study aims to identify whether intracameral lidocaine has effects on the floppy iris syndrome that might develop during surgery.

\section{MATERIAL AND METHOD}

The study was carried out with the permission of Hitit University Medical Faculty Non-interventional Clinical Researchs Ethics Committee (Date: 01.03.2019, Decision No:2019-10). All procedures were carried out in accordance with the ethical rules and the principles of the Declaration of Helsinki. The case-control study conducted retrospectively (via file scan) included 86 cataract patients whom ILA and/ or SLA were applied during phacoemulsification surgery in the eye clinic of our hospital and having no risk factors (patients who did not have a history of use of drugs such as tamsulosin, $\alpha-1$ blockers, 5 - $\alpha$ reductase inhibitors, saw palmetto and some antipsychotic medicine) other than small pupil size for IFIS. The patients systemic diseases (HT, DM, etc.) were noted. Patients' best-corrected visual acuity, biomicroscopic findings, intraocular pressure (IOP) measured with applanation tonometry, and fundus oculus examination findings were recorded. The patients' cataracts were classified according to the Lens Opacities Classification System III (19). In our study, the potential of the two anesthesia types to perform IFIS was compared with each other.

The patients who had a history of prostate disease and therefore used $\alpha$ - adrenergic antagonist, who had over the $3.00 \mathrm{D}$ refractive error, who had white cataract, who had grade $\geq 3$ nuclear cataract and traumatic cataract (as the total surgery durations of these patients are long, they are at a higher risk of developing myosis during surgery), who had pseudoexfoliation, who had a uveitis findings history, and who had posterior synechia in iris were excluded from the study.
Preoperative dilatation of the patients was enhanced using $1 \%$ cyclopentolate, $2.5 \%$ phenylephrine and $1 \%$ tropicamide dripped 3 times in 10-minute intervals (triple dilatation is routinely performed in our clinic to ensure rapid and long-term dilatation). Preoperative pupil diameters were measured with a caliper with an operating microscope. Topical anesthesia of both groups was done with $0.5 \%$ proparacaine. The operations were performed in the same center, by two experienced surgeons (TŞ,SIK). The surgeons randomly chosed the anesthesia type.

The patients involved in the study were divided into two groups. After the site-port of 45 patients was performed, they were administered $0.3-0.5 \mathrm{ml} 1 \%$ unpreserved lidocaine in the anterior chamber. After the 41 patients in the second group were administered proparacaine, they were given $2 \%$ lidocaine $0.5 \mathrm{ml}$ with a 27 gauge injector from the upper nasal to under conjunctiva. Subconjunctival anesthesia is performed as $0.5 \mathrm{ml}$ lidocaine injection with a 27 gauge needle from $5 \mathrm{~mm}$ posterior of the limbus to superior conjunctiva. The type of anesthesia performed during surgery was chosen randomly. The wound site was pressed with cigarette sponge for 1 minute and waited to prevent leakage. Before the capsulorhexis, $3 \%$ sodium hyaluronate viscoelastic was used. The phacoemulsification procedure was done using the 'stop and chop' technique (Infinity Alcon System, Alcon). Peristaltic pump was used during the phacoemulsification procedure (to keep the pressure control system more stable). The following stages of surgery utilized $1.4 \%$ sodium hyaluronate viscoelastic. If any IFIS findings reported in the study conducted by Chang et al. (iris billowing, incarceration in the wound site, or progressive myosis) were seen, these patients were accepted to have developed IFIS (7). The stage when IFIS developed was also recorded. The cases that developed myosis during surgery were given $1 \mathrm{~mL}(1: 1000,1 \mathrm{mg} /$ $\mathrm{mL}$ ) epinephrine. Despite this, the cases that continued to have myosis were administered iris retractor if necessary. No mydriatic method was applied until the IFIS was detected. If posterior capsule rupture or vitreous loss were experienced during surgery, these were also recorded.

Statistical method: Statistical analyses of the data obtained in the study was performed using SPSS (Version 22.0, SPSS Inc., Chicago, IL, USA) package program. In line with the data distribution, descriptive statistics were reported as median (minimum-maximum) and mean \pm standard deviation for the continuous variables. Descriptive statistics of the categorical data were presented as numbers and percentages (\%). The normality distribution for the statistical test choice was assessed using the Shapiro-Wilk test. A comparison of 
the independent two groups measurements was done using the non-parametric Mann Whitney $U$ test as the data did not distribute normally. Relationships and ratio comparisons between the categorical variables were done using Chi-square test. Statistical significance was taken as $\mathrm{p}<0.05$.

Power analysis: Power analysis results indicated $80 \%$ power $(1-\beta=0.80)$ and $\alpha=0.05$ margin of error $\quad(95 \%$ confidence interval) according to 0.335 medium effect size calculated using the Cohen Criteria; 86 patients were involved in the study to identify the statistically significant differences in terms of the ratio of IFIS development in the intracameral lidocaine and subconjunctival lidocaine groups.

\section{RESULTS}

Of all the patients involved in the study, 49 were males (57\%) and 37 were females (43\%). The age range was between 37 and 85 . The average age of the patients was $65.93 \pm 9.21$. No significant differences were found between the groups in terms of the average age (intracameral $63.80 \pm 9.01$, subconjunctival $63.88 \pm 9.09 ; \mathrm{p}>0.05$ ). The number of patients with hypertension was equal in both groups (as it is known that hypertension could cause IFIS) (6). Pre-operative dilate pupil diameters were between $4 \mathrm{~mm}$ and $8.3 \mathrm{~mm}$. While the beginning pupil diameter was $6.21 \pm 0.92 \mathrm{~mm}$ in the intracameral group, it was $6.43 \pm 0.84 \mathrm{~mm}$ in the subconjunctival group. In the beginning, there were no significant differences between the groups in terms of pupil diameters $(\mathrm{p}=0.160)$.

No statistically significant relationships were found in the IFIS development ratios according to the anesthesia type $(\mathrm{p}=0.084)$. IFIS development ratios between the groups were compared in Table 1.

Pupil diameters of those who developed IFIS were lower in both anesthesia groups compared to those who did not; however, this difference was not statistically significant $(\mathrm{p}=0.093, \mathrm{p}=0.116)$. (Table 2, Comparison of the IFIS prevalence according to the pupil diameters).

When the pupil diameter of $6.5 \mathrm{~mm}$ was accepted as the threshold value, a significant increase was found in the IFIS prevalence in patients who had pupil smaller than $6.5 \mathrm{~mm}(\mathrm{p}=0.011)$ (Table 3). IFIS prevalence increased even more in those who had a pupil diameter of less than $6 \mathrm{~mm}(\mathrm{p}=0.009)$ (Table 4).

None of the patients experienced capsule perforation. The cases that demonstrated pupil incarceration or myosis in the wound site during surgery were given epinephrine and $1.4 \%$ viscoelastic. These patients were administered adequate dilation again. None of the patients needed to receive iris retractor.

\begin{tabular}{|c|c|c|c|}
\hline Groups & $\begin{array}{c}\text { Intracameral } \\
(n=45)\end{array}$ & $\begin{array}{l}\text { Subconjunctival } \\
(\mathrm{n}=41)\end{array}$ & P value \\
\hline IFIS & & & 0.084 \\
\hline Yes & $15(33.7 \%)$ & $7(17.1 \%)$ & \\
\hline No & $30(66.7 \%)$ & $34(82.9 \%)$ & \\
\hline
\end{tabular}

Table 2. Comparison of the intraoperative floppy-iris syndrome (IFIS) prevalence according to the pupil diameters

\begin{tabular}{|lcccc|}
\hline Groups & $\mathbf{N}$ & $\begin{array}{c}\text { Mean } \pm \text { SD } \\
(\mathbf{m m})\end{array}$ & $\begin{array}{c}\text { Median }(\mathbf{m i n}- \\
\mathbf{m a x})(\mathbf{m m})\end{array}$ & P value $^{\mathbf{a}}$ \\
\hline $\begin{array}{l}\text { Intracameral } \\
\quad\end{array}$ & & & & 0.093 \\
$\quad$ Yes & 15 & $5.95 \pm 0.59$ & $6(5-7)$ & \\
$\quad$ No & 30 & $6.34 \pm 1.03$ & $6.25(4-8.3)$ & \\
Subconjunctival & & & & 0.116 \\
$\quad$ Yes & 7 & $6.07 \pm 0.61$ & $6(5-7)$ & \\
$\quad$ No & 34 & $6.50 \pm 0.87$ & $6.55(4-7.5)$ & \\
\hline${ }^{a}$ Mann Whitney U test & & & & \\
\hline
\end{tabular}

Table 3. The relationship between the pupil diameter and intraoperative floppy-iris syndrome (IFIS) development when the $6,5 \mathrm{~mm}$ pupil diameter was accepted as the threshold value.

\begin{tabular}{|lccccc|} 
& & \multicolumn{2}{c}{ IFIS } & \multirow{2}{*}{ Total } & P value \\
\cline { 2 - 4 } & & No & Yes & & $0.011^{*}$ \\
\hline Pupil & & & & & \\
$\leq 6.5 \mathrm{~mm}$ & $\mathrm{n}$ & 36 & 19 & 55 & \\
& $\%$ & 65.5 & 34.5 & 100 & \\
$>6.5 \mathrm{~mm}$ & $\mathrm{n}$ & 28 & 3 & 31 & \\
& $\%$ & 90.3 & 9.7 & 100 & \\
\hline${ }^{*}$ Chi-square test statistically significant $(\mathrm{p}<0.05)$ & & & \\
\hline
\end{tabular}

Table 4. The relationship between the pupil diameter and intraoperative floppy-iris syndrome (IFIS) development when the 6 $\mathrm{mm}$ pupil diameter was accepted as the threshold value.

\begin{tabular}{|c|c|c|c|c|c|}
\hline & \multicolumn{2}{|c|}{ IFIS } & \multirow{2}{*}{ Total } & \multirow{2}{*}{$P$ value } \\
\hline & & No & Yes & & \\
\hline Pupil & & & & & $0.009^{*}$ \\
\hline \multirow{2}{*}{$\leq 6 \mathrm{~mm}$} & $\mathrm{n}$ & 26 & 16 & 42 & \\
\hline & $\%$ & 61.9 & 38.1 & 100 & \\
\hline \multirow{2}{*}{$>6 \mathrm{~mm}$} & $\mathrm{n}$ & 38 & 6 & 31 & \\
\hline & $\%$ & 86.4 & 13.6 & 100 & \\
\hline
\end{tabular}

\section{DISCUSSION}

To the best of our knowledge, this study is the first study in the literature that investigated the effect of intracameral lidocaine administered during surgery on the floppy iris syndrome. In comparison to the subconjunctival lidocaine group, IFIS was found to be more common at a proportion of $16.6 \%$ in the group that was given intracameral lidocaine.

Lidocaine is known to prevent the development of the action potential through the inhibition of fast sodium channels in the cell membrane and cause mydriasis independently of the sympathetic and parasympathetic effect (20). Mydriatic effect of lidocaine varies according 
to the way it is administered and the amount of injection (21). To prevent the IFIS development during surgery in patients who used tamsulosin, a study conducted before administered intracameral lidocaine- epinephrine to a group of patients and administered nothing in the anterior chamber to another group of patients. IFIS development ratio was $38.5 \%$ in the group that was administered lidocaine-epinephrine and $25.5 \%$ in the other group. Contrary to what was expected, IFIS was found to be higher in the group that was administered lidocaine - epinephrine. This finding was considered to result from the smaller beginning pupil diameter in the group that was administered lidocaine- epinephrine (22). Another study conducted with patients who used tamsulosin and received cataract surgery administered sub-Tenon's lidocaine (2\%) to one group and intracameral lidocaine (1\%) to the other group. The group that was administered sub-Tenon's lidocaine was found to develop IFIS at a proportion of $8.8 \%$, while this ratio was $48.6 \%$ in the group administered intracameral lidocaine. It was concluded that this finding resulted from the longer effect of sub-Tenon's lidocaine due to its accumulation around the eyeball and longer maintenance of mydriasis (23). Savino et al. (20) gave sub-Tenon's ropivacaine to a group of patients who received strabismus surgery and sub-Tenon's saline accompanied by general anesthesia to the other group. The group that was given sub-Tenon's ropivacaine was found to develop mydriasis approximately 5 minutes later. This effect was found to occur as local anesthesia (ropivacaine) caused a blockage in ciliary ganglion and ciliary nerves. Lidocaine amount decreases in a short time in intracameral anesthesia due to high liquid flow in the anterior chamber and viscoelastic substance administration. Mydriatic effect of intracameral lidocaine is known to end within 10-15 minutes (24). We observed that IFIS findings were more common in patients who underwent intracameral anesthesia in our cataract surgeries. In order to understand whether there is a difference between anesthesia types, we excluded the factors (tamsulosin use, ...) that may affect IFIS in our study. We think that the reason for intracameral lidocaine to cause IFIS could be explained with this possible mechanism: lidocaine given to the anterior chamber inhibits both circular and meridional fibers of iris, which decreases the parasympathetic system activities dominant in eyes and develops mydriasis. However, tonus of the iris decreases depending on the inhibition of two muscle groups, and iris becomes flask. In this case, the iris becomes more sensitive against fluid flow and manipulations in the anterior chamber, and floppy iris syndrome findings might occur. We thought that this might also be meaningful, but we could not obtain a meaningful result in our study.
In our study, we observed that the IFIS ratio increased as the pupil diameter decreased. However, the difference was not statistically significant. IFIS development was more common in pupil diameters of less than $6.5 \mathrm{~mm}$, and the difference between them was significant. As to the pupil diameters of less than $6 \mathrm{~mm}$, the prevalence of IFIS development was even more. Casuccio et al. (25) reported that a small pupil diameter was a good indicator of IFIS development. In their study that was conducted later, Chen et al. (22) detected that preoperative small pupil diameter (less than $6.5 \mathrm{~mm}$ was accepted as small) increased the probability of IFIS development during surgery. It was reported that IFIS might have developed because the iris surface area was more sensitive against the liquid flow in the anterior chamber as the pupil diameter gets smaller. Our study also confirmed that small pupil diameter is a risk factor for IFIS.

This study has some limitations. First, our study was retrospective. Secondly, before the study, a power analysis was performed for the number of patients to be involved in the study. However, we think that prospective studies to be conducted with a higher number of patients would yield more meaningful results.

\section{CONCLUSION}

While performing cataract surgery, patients with small pupil diameters should be careful about the development of IFIS. The effect of intramaral lidocaine use on IFIS is controversial. Future prospective studies to be conducted with a higher number of cases could be a guide on this issue.

\section{ETHICAL DECLARATIONS}

Ethics Committee Approval: The study was carried out with the permission of Hitit University Medical Faculty Non-interventional Clinical Researchs Ethics Committee (Date: 01.03.2019, Decision No: 2019-10).

Informed Consent: Because the study was designed retrospectively, no written informed consent form was obtained from patients.

Referee Evaluation Process: Externally peer-reviewed.

Conflict of Interest Statement: The authors have no conflicts of interest to declare.

Financial Disclosure: The authors declared that this study has received no financial support.

Author Contributions: All of the authors declare that they have all participated in the design, execution, and analysis of the paper, and that they have approved the final version. 


\section{REFERENCES}

1. Yan W, Wang W, van Wijngaarden P, Mueller A, He M. Longitudinal changes in global cataract surgery rate inequality and associations with socioeconomic indices. Clin Exp Ophthalmol 2019; 47: 453-60.

2. Shah R. Anesthesia for cataract surgery: Recent trends. Oman J Ophthalmol 2010; 3: 107-8.

3. Ezra DG, Allan BD. Topical anaesthesia alone versus topical anaesthesia with intracameral lidocaine for phacoemulsification. Cochrane Database Syst Rev 2007; CD005276.

4. Tan CS, Fam HB, Heng WJ, Lee HM, Saw SM, Au Eong KG. Analgesic effect of supplemental intracameral lidocaine during phacoemulsification under topical anaesthesia: a randomised controlled trial. Br J Ophthalmol 2011; 95: 837-41.

5. Anderson CJ. Subconjunctival anesthesia in cataract surgery. J Cataract Refract Surg 1995; 21: 103-5.

6. Neff KD, Sandoval HP, Fernández de Castro LE, Nowacki AS, Vroman DT, Solomon KDl. Factors associated with intraoperative floppy iris syndrome. Ophthalmology 2009; 116: 658-63.

7. Chang DF, Campbell JR. Intraoperative floppy iris syndrome associated with tamsulosin. J Cataract Refract Surg 2005; 31: 664-73.

8. Cheung CM, Awan MA, Sandramouli S. Prevalence and clinical findings of tamsulosin-associated intraoperative floppy-iris syndrome. J Cataract Refract Surg 2006; 32: 1336-9.

9. Takmaz T, Can I. Clinical features, complications, and incidence of intraoperative floppy iris syndrome in patients taking tamsulosin. Eur J Ophthalmol 2007; 17: 909-13.

10. Oshika T, Ohashi Y, Inamura M, et al. Incidence of Intraoperative Floppy Iris Syndrome in Patients on Either Systemic or Topical a1-Adrenoceptor Antagonist. Am J Ophthalmol 2007; 143: 150-1.

11.Issa SA, Dagres E. Intraoperative floppy-iris syndrome and finasteride intake. J Cataract Refract Surg 2007; 33: 2142-3.

12. Goepel M, Hecker U, Krege S, Rübben H, Michel MC. Saw palmetto extracts potently and noncompetitively inhibit human alpha1-adrenoceptors in vitro. Prostate 1999; 38: 208-15.

13.Ünal M, Yücel İ, Tenlik A. Intraoperative floppy-iris syndrome associated with chronic use of chlorpromazine. Eye 2007; 21: 1241-2.

14. Pringle E, Packard R. Antipsychotic agent as an etiologic agent of IFIS. J Cataract Refract Surg 2005; 31: 2240-1.

15. Chang DF, Braga-Mele R, Mamalis N, et al. ASCRS Cataract Clinical Committee. ASCRS White Paper: Clinical review of intraoperative floppy-iris syndrome. J Cataract Refract Surg 2008; 34: $2153-62$

16. Chang DF, Osher RH, Wang L, Koch DD. Prospective multicenter evaluation of cataract surgery in patients taking tamsulosin (Flomax). Ophthalmology 2007; 114: 957-64.

17. Manvikar S, Allen D. Cataract surgery management in patients taking tamsulosin. J Cataract Refract Surg 2006; 32: 1611-4.

18. Goldman JM, Karp CL. Adjunct devices for managing challenging cases in cataract surgery: capsular staining and ophthalmic viscosurgical devices. Curr Opin Ophthalmol 2007; 18: 52-7.

19. Chylack LT, Wolfe JK, Singer DM, et al. The lens opacities classification system III. the longitudinal study of cataract study group. Arch Ophthalmol 1993; 111: 831-6.

20. Savino G, Perrotta V, Colucci D, et al. Mydriasis induced by subTenon's ropivacaine injection in patients undergoing strabismus surgery. J AAPOS 2010; 14: 124-6.

21.Lincoff $\mathrm{H}$, Zweifach $\mathrm{P}$, Brodie $\mathrm{S}$, et al. Intraocular injection of lidocaine. Ophthalmology 1985; 92: 1587-91.

22. Chen AA, Kelly JP, Bhandari A, Wu MC. Pharmacologic prophylaxis and risk factors for intraoperative floppy-iris syndrome in phacoemulsification performed by resident physicians. J Cataract Refract Surg 2010; 36: 898-905.
23. Klysik A, Korzycka D. Sub-Tenon injection of $2 \%$ lidocaine prevents intra-operative floppy iris syndrome (IFIS) in male patients taking oral $\alpha$-adrenergic antagonists. Acta Ophthalmol 2014; 92: 535-40.

24. Oberg TJ, Sikder S, Jorgensen AJ, Mifflin MD. Topicalintracameral anesthesia without preoperative mydriatic agents for Descemet-stripping automated endothelial keratoplasty and phacoemulsification cataract surgery with intraocular lens implantation. J Cataract Refract Surg 2012; 38: 384-6.

25.Casuccio A, Cillino G, Pavone C, Spitale E, Cillino S. Pharmacologic pupil dilation as a predictive test for the risk for intraoperative floppy-iris syndrome. J Cataract Refract Surg 2011; 37: 1447-54. 